\title{
Associação entre tempo de internação e evolução do estado nutricional de crianças internadas em um hospital universitário
}

\section{Association between length of hospital stay and evolution of nutritional status of children admitted to a university hospital}

Emilia A. de Magalhães'

Marcela A. L. P. Martins ${ }^{2}$

Claudia C. Rodrigues ${ }^{3}$

Annie S. B. Moreira ${ }^{3}$

1 Pós-graduada em Nutrição e Pediatria, Universidade Gama Filho. Rio de Janeiro, RJ, Brasil.

${ }^{2}$ Nutricionista do Hospital Universitário Pedro Ernesto. Rio de Janeiro, RJ, Brasil.

${ }^{3}$ Professora adjunta, Departamento de Nutrição Aplicada, Instituto de Nutrição, Universidade do Estado do Rio de Janeiro. Rio de Janeiro, RJ, Brasil.

Correspondência / Correspondence

Emilia A. de Magalhães

E-mail:emilia.magalhaes@yahoo.com.br

\section{Resumo}

Objetivo: Avaliar o estado nutricional de crianças hospitalizadas e identificar os fatores de risco associados com o tempo de internação hospitalar. Métodos: Estudo longitudinal, realizado com todas as crianças de 0 a 10 anos $(n=200)$ internadas em um hospital universitário entre fevereiro e dezembro de 2011. Foram coletados dados de identificação, doença, presença de anemia e tempo de internação. Para avaliação do estado nutricional, utilizou-se o escore z para os indicadores de P/I, E/I, P/E e IMC/I. Utilizou-se o programa SPSS versão 17 para análise dos dados, considerando significante $\mathrm{p}<0,05$. Resultados: Do total de crianças acompanhadas, $56 \%$ eram do sexo masculino e a média da idade foi de $53,28 \pm 47,65$ meses. Verificou-se que as principais condições associadas ao maior tempo de internação foram faixa etária dos lactentes, diagnóstico de doença hematológica, baixo-peso e presença de anemia na internação. A manutenção do peso durante a hospitalização foi fator associado ao menor tempo de internação. Conclusões: Verificou-se que o baixo-peso na internação, a presença de anemia e a faixa etária do lactente foram os fatores determinantes de maior tempo de internação. Assim, o acompanhamento do estado nutricional das crianças hospitalizadas foi fundamental para o maior entendimento em relação aos fatores associados ao maior tempo de hospitalização, possibilitando melhor abordagem para manutenção e/ou recuperação do estado nutricional.

Palavras-chave: Estado Nutricional. Tempo de Internação. Internação Hospitalar. Desnutrição. 


\section{Abstract}

Objective: To evaluate the nutritional status of hospitalized children and to identify the risks factors associated with length of hospital stay. Methods: A longitudinal study of 200 children aged 0-10 years, admitted to a university hospital from February to December 2011. Data were collected for identification, disease, anemia and length of hospital stay. To evaluate the nutritional status, the $\mathrm{z}$ score was used for W/A, H/A, W/H and BMI/A indicators. Statistical analysis was carried out through SPSS version 17 software, being significant $\mathrm{p}<0.05$. Results: $56 \%$ were male and the average age was $53.28 \pm 47.65$ months. The main conditions associated with a longer length of hospital stay were the infants' age group, the diagnosis of hematologic disease, low weigh and anemia on admission. Weight maintenance during hospitalization was a factor associated to shorter length hospital stay. Conclusions: It was verified that underweight on admission, anemia and infants' age group were the determinants of a longer length of hospital stay. Then, the follow-up of nutritional status of hospitalized children was fundamental to greater understanding about the factors associated with longer hospital stay, allowing a better approach to maintenance and/or recovery of nutritional status.

Key words: Nutritional Status. Length of Stay. Hospitalization. Malnutrition.

\section{Introdução}

Apesar de nas últimas décadas ter havido expressiva redução na prevalência de desnutrição energético-proteica (DEP) em crianças menores de cinco anos no Brasil e no mundo, ${ }^{1}$ essa doença continua a ser um relevante problema de saúde pública no país, principalmente nas regiões Norte e Nordeste, na área rural e nos bolsões de pobreza das periferias das grandes metrópoles, com consequências graves no crescimento, desenvolvimento e sobrevida das crianças. ${ }^{2}$

Cerca de $80 \%$ das crianças desnutridas residem na Ásia, $15 \%$ na África e 5\% na América Latina, sendo que 43\% delas (230 milhões) são desnutridas crônicas. ${ }^{3}$ Além disso, os índices de letalidade por desnutrição grave em tratamento hospitalar permanecem elevados. ${ }^{1}$ De acordo com dados da Organização Pan-Americana da Saúde (OPAS), a 
cada ano, mais de 200 mil crianças morrem antes dos cinco anos nas Américas, devido a desnutrição e doenças preveníveis. Dentre as crianças com desnutrição grave, 20 a $30 \%$ delas vão a óbito durante tratamento nos serviços de saúde desses países. ${ }^{3}$

Existem diversos estudos na literatura sobre prevalência da desnutrição em crianças, mas infelizmente não é de rotina no atendimento hospitalar a prática da avaliação do estado nutricional e seu seguimento durante a internação, dificultando o diagnóstico e o tratamento dos agravos nutricionais e contribuindo para a ocorrência de complicações e hospitalizações prolongadas. ${ }^{1,3}$ É de suma importância conhecer e acompanhar o estado nutricional de crianças hospitalizadas, para melhor compreender os fatores relacionados ao maior tempo de internação hospitalar e, com isso, melhorar o manejo da terapia nutricional para manutenção e/ou recuperação do estado nutricional do paciente. ${ }^{3}$ Assim sendo, o objetivo deste estudo foi avaliar o estado nutricional das crianças hospitalizadas e identificar os fatores de risco associados com o tempo de internação hospitalar.

\section{Métodos}

Estudo longitudinal realizado com todas as crianças de zero a dez anos ( $n=200)$, de ambos os sexos, internadas na enfermaria de Pediatria do Hospital Universitário Pedro Ernesto (HUPE), localizado na cidade do Rio de Janeiro, no período de fevereiro a dezembro de 2011. O período de internação variou de um a 120 dias. Foram excluídas da amostra crianças maiores que dez anos. O estudo obteve aprovação do Comitê de Ética do HUPE, sob registro 102.652.

Todos os dados dos pacientes foram coletados dos prontuários. Foram coletados os dados da internação: nome, prontuário, data da internação, tempo de internação, data de nascimento, idade, prematuridade, sexo, diagnóstico, comorbidades, exame laboratorial (hemoglobina), presença de anemia (quando hemoglobina menor que $11,5 \mathrm{~g} / \mathrm{dL}$ ); dados da alimentação durante o período da internação: utilização de nutrição enteral (NE), uso de suplementação via oral (SVO), dieta zero; dados da avaliação nutricional na admissão e na alta: peso, estatura, peso para idade (P/I), estatura para idade (E/I), peso para estatura (P/E), índice de massa corporal para idade (IMC/I) e classificação do estado nutricional. Além disso, realizou-se o escore de risco nutricional, utilizando dados da admissão: perda de peso maior que $2 \%$, ingestão alimentar menor que $50 \%$ e presença de dor.

Com relação à evolução do estado ponderal, considerou-se a diferença entre o peso da alta e o da admissão, para determinar ganho ou perda ponderal durante a hospitalização. 
As doenças foram estratificadas em cinco grupos: doença respiratória, hematológica, digestiva, infecciosa grave e outras (por exemplo, doenças renais, diabetes e doenças metabólicas). A classificação do estado nutricional foi realizada usando o escore z para os indicadores de P/I, E/I, P/E e IMC/I (na internação e na alta), tendo como padrão de referência a curva proposta pela Organização Mundial da Saúde (OMS). ${ }^{4}$ Considerou-se eutrofia quando o escore $\mathrm{z}$ de um dos indicadores (P/I, E/I, P/E e IMC/I) estava entre +1 e -2; baixo-peso quando o escore z de um dos indicadores ia de -2 a -3; e muito baixo-peso quando escore z de um dos indicadores era menor que -3 , segundo a classificação da OMS. ${ }^{5}$

As crianças classificadas como baixo-peso e muito baixo-peso foram agrupadas como baixo-peso. Quanto às classificadas como risco de sobrepeso, sobrepeso, obesidade e obesidade grave, consideraram-se os critérios do SISVAN 2008: para crianças de 0 a 5 anos incompletos, $\mathrm{P} / \mathrm{E}$ e/ou IMC/I, escore $\mathrm{z}>+1 \mathrm{e} \leq+2$, classificadas como risco de sobrepeso; $>+2$ e $\leq+3$ como sobrepeso; e $>+3$ como obesidade; e para crianças de 5 a 10 anos incompletos, IMC/I escore $\mathrm{z}>+1$ e $\leq+2$ classificadas como sobrepeso; > +2 e $\leq+3$ como obesidade; e $>+3$ como obesidade grave, ${ }^{6}$ sendo agrupadas como excesso de peso.

Os dados antropométricos da internação foram coletados até 48 horas da admissão, e os dados da alta até 48 horas antes da ocasião da alta. As crianças menores de dois anos foram pesadas sem roupas em balança digital do tipo pesa-bebê, com capacidade de 16 $\mathrm{kg}$ e sensibilidade de $10 \mathrm{~g}$, e seu comprimento aferido em decúbito dorsal sobre uma superfície plana, com a utilização de régua antropométrica, com escala em centímetros, até o máximo de $1 \mathrm{~m}$, sempre com a ação conjunta de dois examinadores para garantir o posicionamento da cabeça e afixação de ambos os pés. As crianças acima de dois anos foram pesadas com o mínimo de roupa, em balança tipo adulto, com precisão de 100 g. A altura foi aferida com a criança em pé, encostada numa régua vertical com escala métrica, em centímetros, medindo até $150 \mathrm{~cm}$ e afixada na parede. ${ }^{7}$

Estratificou-se o estado nutricional da população de acordo com o diagnóstico (respiratório, hematológico, digestivo, infeccioso grave e outros), sendo comparado o tempo de internação com as variáveis (sexo, idade, prematuridade, diagnóstico, estado nutricional, mudança de peso, presença de anemia, ingestão alimentar menor que 50\% - inadequada, presença de dor, uso de NE, uso de SVO, dieta zero). Além disso, foram avaliados os fatores de risco para tempo de internação maior que nove dias, e analisou-se a evolução do estado nutricional de acordo com o escore z de P/I, E/I, P/E e IMC/I na admissão e na alta, e de acordo com crianças desnutridas e não desnutridas, na admissão e na alta. 
Para análise estatística dos resultados, utilizou-se o programa SPSS versão 17. Os dados foram apresentados como média +- desvio padrão e frequência. Utilizou-se o teste qui-quadrado para testar variáveis categóricas independentes (para avaliação do estado nutricional de acordo com o diagnóstico), utilizaram-se os testes de Mann Whitney e Kruskal-Wallis, testes não paramétricos para comparar as médias de variáveis contínuas (comparação do tempo de internação com as variáveis clínicas). Utilizaram-se odds ratio (OR) e intervalo de confiança (IC) para a avaliação dos fatores de risco com tempo de internação prolongado (maior do que nove dias) e usou-se teste $t$ pareado (variáveis contínuas com distribuição normal) e Wilcoxon (sem distribuição normal) para a avaliação da evolução do estado nutricional. Em todos os casos, considerou-se estatisticamente significante quando $\mathrm{p}<0,05$.

\section{Resultados}

As características dos pacientes estão descritas na tabela 1. Do total de crianças acompanhadas, $56 \%$ eram do sexo masculino e a média de idade foi 53,28 $\pm 47,65$ meses, sendo 51 lactentes, 43 pré-escolares e 64 escolares. Quanto ao estado nutricional, 18,8\% das crianças eram de baixopeso, $58,7 \%$ eutróficas e 22,5\% com excesso de peso. O percentual de anemia foi de $54,8 \%$ das crianças. As crianças com baixo-peso eram mais novas que as com peso normal (27,5+-38,4 meses; $61,2+-47,8$ meses; $\mathrm{p}<0,01)$.

O diagnóstico mais frequente foi de doença respiratória, apresentando 29,5\%, seguido de $12,5 \%$ com doença hematológica, $11 \%$ com processo infeccioso grave e 9,5\% com doença do trato digestivo. Verificou-se que, nas primeiras 24 horas de internação, 10,2\% ingeriram menos que $50 \%$ das necessidades nutricionais e 18,1\% queixavam-se de dor. Em relação ao uso de suporte nutricional durante a internação, 10,1\% utilizaram nutrição enteral, 9,5\% usaram suplemento via oral e $19,5 \%$ ficaram em dieta zero por algum momento. 
Tabela 1. Características dos pacientes de acordo com as variáveis estudadas. Rio de Janeiro,RJ, 2011.

\begin{tabular}{lccc}
\hline \multicolumn{1}{c}{ Variáveis } & $\mathrm{n}$ Total & $\mathrm{n}$ & $\%$ \\
\hline Sexo (Masculino, "n" 112) & 200 & 112 & $56,0 \%$ \\
Prematuro & 157 & 35 & $22,3 \%$ \\
Estado nutricional & 138 & & \\
$\quad$ Baixo-peso & & 26 & $18,8 \%$ \\
$\quad$ Eutrófico & & 81 & $58,7 \%$ \\
$\quad$ Excesso de peso & 200 & 31 & $22,5 \%$ \\
Diagnóstico & & & \\
$\quad$ Respiratório & & 59 & $29,5 \%$ \\
$\quad$ Hematológico & & 25 & $12,5 \%$ \\
$\quad$ Digestivo & & 19 & $9,5 \%$ \\
$\quad$ Infeccioso grave & & 22 & $11,0 \%$ \\
$\quad$ Outros & & 75 & $37,5 \%$ \\
Anemia & 115 & 63 & $54,8 \%$ \\
Ingestão <50\% & 94 & 21 & $10,2 \%$ \\
Dor & 94 & 17 & $18,1 \%$ \\
Usou NE & 200 & 20 & $10,1 \%$ \\
Usou SVO & 200 & 19 & $9,5 \%$ \\
Dieta zero & 200 & 39 & $19,5 \%$ \\
\hline
\end{tabular}

Verificou-se que as principais condições associadas ao maior tempo de internação foram diagnóstico de doença hematológica, presença de anemia e baixo-peso na admissão. A manutenção de peso na internação foi um fator associado ao menor tempo de internação, quando comparado aos que perderam e aos que ganharam peso. Sexo, prematuridade, adequação de ingestão dietética e presença de dor não tiveram associação com o tempo de internação (tabela 2). A tabela 2 compara o tempo de internação com as variáveis estudadas: sexo, idade, prematuridade, diagnóstico, estado nutricional, mudança de peso, presença de anemia, ingestão alimentar menor que 50\% (inadequada) e presença de dor, utilizando os testes de Man Witney e Kruskal-Wallis. 
Tabela 2. Comparação entre o tempo de internação hospitalar em relação às variáveis estudadas. Rio de Janeiro, RJ, 2011.

\begin{tabular}{|c|c|c|c|c|}
\hline & \multicolumn{3}{|c|}{ Tempo de internação hospitalar } & \multirow[b]{2}{*}{$\mathrm{p}$} \\
\hline & $\mathrm{N}$ & Média & $\mathrm{EP}$ & \\
\hline \multicolumn{5}{|l|}{ Sexo } \\
\hline Feminino & 88 & 8,49 & 2,05 & \\
\hline Masculino & 112 & 8,20 & 0,89 & $0,32(\mathrm{a})$ \\
\hline \multicolumn{5}{|l|}{ Idade } \\
\hline Lactente & 63 & 9,84 & 1,47 & \\
\hline Pré-escolar & 65 & 5,84 & 1,21 & \\
\hline Escolar & 72 & 8,78 & 1,98 & $0,07(b)$ \\
\hline \multicolumn{5}{|l|}{ Prematuro } \\
\hline Não & 108 & 8,98 & 1,37 & \\
\hline Sim & 28 & 8,21 & 1,51 & $0,91(a)$ \\
\hline \multicolumn{5}{|l|}{ Diagnóstico } \\
\hline Respiratório & 48 & 8,40 & 0,93 & \\
\hline Hematológico & 20 & 11,95 & 3,01 & \\
\hline Digestivo & 14 & 6,43 & 1,82 & \\
\hline Infeccioso grave & 19 & 9,16 & 3,11 & \\
\hline Outros & 57 & 7,18 & 2,13 & $0,02 *(b)$ \\
\hline \multicolumn{5}{|l|}{ Estado nutricional } \\
\hline Baixo-peso & 20 & 14,25 & 4,19 & \\
\hline Eutrófico & 67 & 7,22 & 0,67 & \\
\hline Excesso de peso & 29 & 8,55 & 2,15 & $0,006^{*}(\mathrm{~b})$ \\
\hline \multicolumn{5}{|l|}{ Mudança de peso } \\
\hline Não mudou & 9 & 3,56 & 1,25 & \\
\hline Ganhou peso & 58 & 10,26 & 1,11 & \\
\hline Perdeu peso & 41 & 12,85 & 3,21 & $0,002 *(b)$ \\
\hline \multicolumn{5}{|l|}{ Anemia } \\
\hline Normal & 47 & 8,15 & 1,46 & \\
\hline Anemia & 51 & 13,18 & 2,56 & $0,037 *(a)$ \\
\hline \multicolumn{5}{|l|}{ Na admissão } \\
\hline Ingestão adequada & 70 & 10,31 & 1,35 & \\
\hline Ingestão inadequada & 18 & 12,83 & 6,36 & $0,62(\mathrm{a})$ \\
\hline Sem dor & 71 & 11,56 & 2,00 & \\
\hline Dor & 17 & 7,76 & 2,19 & $0,54(\mathrm{a})$ \\
\hline
\end{tabular}

(a) Man Witney; (b) Kruskal-Wallis * $\mathrm{p}<0,05$ 
Observou-se que os fatores com probabilidade de internação mais longa que nove dias foram a faixa etária dos lactentes (0-1 ano) (OR 2,3), presença de desnutrição na internação (OR 6,28) e presença de anemia na admissão (OR 3,68), segundo a tabela 3. A tabela 3 apresenta os fatores de risco associados a um tempo de internação mais longo (maior que 9 dias) através de análise univariada, utilizando odds ratio e intervalo de confiança.

Tabela 3. Fatores de risco para tempo de internação maior que nove dias. Análise univariada (n=180). Rio de Janeiro, RJ, 2011.

\begin{tabular}{lcccc}
\hline & \multicolumn{2}{c}{ OR } & - & \multicolumn{2}{c}{ IC } \\
\hline Lactente & 2,30 & 1,12 & - & $189^{*}$ \\
Desnutrição & 6,28 & 2,18 & - & $88^{*}$ \\
Anemia & 3,68 & 1,54 & - & $8,79 *$ \\
\hline
\end{tabular}

$\mathrm{OR}=$ odds ratio $\mathrm{IC}=$ intervalo de confiança $* \mathrm{p}<0,05$

Em relação à evolução do estado nutricional, não houve diferença na média do escore z quando se comparou o peso na internação e na alta. Quando se estratificaram as crianças em baixo-peso e sem baixo-peso, verificou-se melhora importante do estado nutricional, com redução do número de crianças com baixo-peso de 21,6\% para 16,2\% (tabela 4).

Tabela 4. Evolução do estado nutricional de acordo com a classificação de crianças com e sem baixo-peso, na admissão e na alta, utilizando o teste de Wilcoxon Signed Ranks. Rio de Janeiro, RJ, 2011.

\begin{tabular}{lcccc}
\hline & \multicolumn{2}{c}{ Admissão } & \multicolumn{2}{c}{ Alta } \\
& $\mathrm{N}$ & $\%$ & $\mathrm{~N}$ & $\%$ \\
\hline Baixo-peso & 24 & 21,6 & 18 & 16,2 \\
Sem baixo-peso & 87 & 78,4 & 93 & $84,0^{*}$ \\
\hline
\end{tabular}

Teste de Wilcoxon Signed Ranks, *p<0,05 


\section{Discussão}

A desnutrição intra-hospitalar é foco de muitos trabalhos nos últimos anos. A prevalência de desnutrição é alta e varia de $24,8 \%^{8}$ até $56,7 \%^{1}$ e $71,2 \% .^{9}$ Neste trabalho, encontrou-se menor frequência de baixo-peso (18,8\%), seguida de 22,5\% de excesso de peso e $58,7 \%$ de eutrofia. Foram avaliadas 200 crianças com 32\% lactentes, 37\% pré-escolares e 31\% escolares com média de idade de 53,28 477,65 meses. Provavelmente, o percentual de desnutrição nesta pesquisa foi inferior ao de outros estudos, pelo fato de as crianças terem média de idade superior. Os estudos avaliaram o estado nutricional de crianças em diferentes idades variando desde 9,8 meses, ${ }^{10} 10,5$ meses, ${ }^{1} 17$ meses, ${ }^{8}$ 21,6 meses. ${ }^{3}$ Apenas um estudo avaliou os escolares com mediana de sete anos. ${ }^{11}$

Delgado et al. ${ }^{12}$ estratificaram a população em relação ao estado nutricional e verificaram que os desnutridos tiveram média de idade superior (25,6 meses) que os bem nutridos (10,7 meses). De forma interessante, os dados do presente estudo mostraram resultado oposto: as crianças com baixo-peso tinham média de idade inferior quando comparadas às sem baixo-peso, o que reforça a condição idade-lactente como fator de risco para desnutrição.

A frequência de crianças prematuras foi de 22,3\%, próxima à encontrada na literatura, que variou de $13,6 \%^{1}$ a $33,8 \%^{10}$. Já a frequência de anemia na admissão foi de 54,8\%. Estudo de Panato et al. ${ }^{13}$ encontrou prevalência de 70,74\% de anemia ferropriva nas crianças hospitalizadas, bem como Vaccaro, ${ }^{14}$ que encontrou, em um hospital, 92\% nas crianças com idades entre seis e 24 meses e $88 \%$ nos menores de seis meses e, em outro hospital, $97 \%$ e $90 \%$, respectivamente. Sendo assim, os resultados deste estudo confirmam a alta prevalência de anemia em pacientes hospitalizados.

Verificou-se que o diagnóstico mais frequente foi de doença respiratória. Estudo realizado por Silveira et al. ${ }^{15}$ também encontrou o grupo da doença respiratória como sendo o mais frequente, e outros pesquisadores encontraram a pneumonia como o principal diagnóstico. ${ }^{1,3,8,10}$

Verificou-se que as principais condições associadas ao maior tempo de internação foram a faixa etária dos lactentes e o baixo-peso na admissão. Outros estudos, da mesma forma, encontraram que crianças desnutridas permaneciam mais tempo internadas. ${ }^{8,9,11,16}$ Simões et al. ${ }^{11}$ também encontraram maior tempo de internação entre os lactentes, o que provavelmente se deve à maior presença de doenças respiratórias, como pneumonia, nessa faixa etária. De forma original, nossos dados mostraram relação entre tempo de internação de acordo com o diagnóstico (doença hematológica) e presença de anemia também como determinante.

Poucos estudos avaliaram os fatores associados à maior permanência hospitalar. Apenas um estudo encontrou que crianças que evoluíram com perda ponderal permaneceram mais do que nove dias internadas, ${ }^{3}$ e o estudo de Sarni et al. ${ }^{1}$ verificou correlação inversa entre o escore z de 
$\mathrm{P} / \mathrm{E}$ da alta e o tempo de internação em crianças desnutridas e eutróficas. O presente estudo foi pioneiro em estabelecer que, além do estado nutricional, idade e anemia são fatores de risco para maior internação hospitalar. A faixa etária de lactentes aumentou a chance para internação mais longa, com OR 2,3; presença de desnutrição na internação, com OR 6,28; e presença de anemia à admissão, com OR 3,68.

Não se verificou relação do gênero com tempo de internação, apenas alguns trabalhos

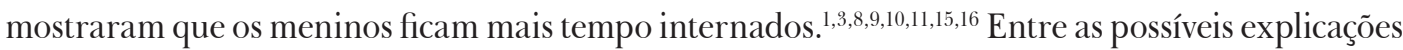
para tal fato estaria a maior exposição a agentes infecciosos e traumas, já que há maior liberdade de ações e brincadeiras se comparado ao gênero feminino, por razões socioculturais em nosso meio. ${ }^{11}$

A frequência de baixo-peso se reduziu durante a internação, mostrando que a terapia nutricional foi eficaz. Sarni et al. ${ }^{10}$ mostraram melhora estatisticamente significante do escore z de P/E e P/I, sendo que outros pesquisadores também encontraram que um grupo de desnutridos teve melhora significativa em relação ao escore z de P/E. Já Ferreira et al. ${ }^{9}$ mostraram redução da ordem de 15,2\% na prevalência da desnutrição grave e de 6,5\% na de desnutrição leve, mas o número de casos de desnutrição moderada aumentou em 24,7\%. Rocha et al. ${ }^{3}$ observaram que as crianças admitidas com desnutrição moderada e grave permaneceram com seu estado nutricional inalterado, e dentre as que eram eutróficas, 9,17\% apresentaram comprometimento nutricional.

\section{Conclusão}

Neste estudo, verificou-se que baixo-peso na internação, faixa etária do lactente e presença de anemia foram fatores determinantes de maior tempo de internação. Assim, o acompanhamento do estado nutricional das crianças hospitalizadas foi fundamental para o maior entendimento dos fatores associados ao maior tempo de internação hospitalar, possibilitando melhor abordagem para a manutenção e/ou recuperação do estado nutricional.

Conhecer os fatores determinantes do tempo de internação permitirá o oferecimento de terapia nutricional adequada e precoce à doença e ao estado nutricional apresentado, colaborando, assim, para a melhora do prognóstico do paciente.

\section{Agradecimentos}

À equipe de nutricionistas do Hospital Universitário Pedro Ernesto, que realizou as avaliações nutricionais e a coleta dos dados dos pacientes, contribuindo assim para a construção deste estudo. 


\section{Referências}

1. Sarni RO, Carvalho MF, do Monte CM, Albuquerque ZP, Souza FI. Anthropometric evaluation, risk factors for malnutrition, and nutritional therapy for children in teaching hospitals in Brazil. J Pediatr (Rio J). 2009;85(3):223-8.

2. Brasil. Ministério da Saúde. Manual de atendimento da criança com desnutrição grave em nível hospitalar. Brasília, DF: Ministério da Saúde; 2005.

3. Rocha GA, Rocha EJ, Martins CV. The effects of hospitalization on the nutritional status of children. J Pediatr (Rio J). 2006;82:70-4.

4. Organização Mundial da Saúde. WHO child growth standards: length/height-for-age, weight-forage, weight-for-length, weight-for-height and body mass index-for-age: methods and development. Geneva: WHO; 2006.

5. Organização Mundial da Saúde. WHO Global Database on Child Growth and Malnutrition. WHO; 2009.

6. Brasil. Ministério da Saúde. Protocolos do Sistema de Vigilância Alimentar e Nutricional - SISVAN na assistência à saúde. Brasília, DF: Ministério da Saúde; 2008.

7. Brasil. Ministério da Saúde. Vigilância alimentar e nutricional -SISVAN: antropometria: como pesar e medir. Brasília, DF: Ministério da Saúde; 2004.

8. De Oliveira AF, Oliveira FL, Juliano Y, Ancona-Lopez F. Nutritional evolution of hospitalized children who were under nutritional orientation. Rev Nutr. 2005;18(3):341-8.

9. Ferreira HS, França AO. Evolution of nutritional status in hospitalized children. J Pediatr (Rio J). 2002;78(6):491-6.

10. Sarni RO, de Souza FI, Catherino P, Kochi C, Oliveira FL, de Nóbrega FJ. Tratamento da desnutrição em crianças hospitalizadas em São Paulo. Rev Assoc Med Bras. 2005;51(2):106-12.

11. Simões AP, Palchetti CZ, Patin RV, Mauri JF, Oliveira FL. Nutritional status of children and adolescents hospitalized at the pediatric surgery unit. Rev Paul Pediatr. 2010;28(1):41-7.

12. Delgado AF, Okay TS, Leone C, Nichols B, Del Negro GM, Vaz FA. Hospital malnutrition and inflammatory response in critically ill children and adolescents admitted to a tertiary intensive care unit. Clinics. 2008;63:357-62.

13. Panato CS, Denardi GT, Nozaki VT. Prevalência de anemia ferropriva e consumo de ferro em crianças hospitalizadas. Revista Saúde e Pesquisa. 2011;4(1):45-50.

14. Vaccaro AM. Análise da condução da anemia em crianças menores de 5 anos, internadas em dois hospitais, São Paulo [tese]. São Paulo (SP): Escola Paulista de Medicina da Universidade Federal de São Paulo; 2001. 
15. Silveira CR, de Mello ED, Carvalho PR. Evolution of nutritional status of pediatric in patients of a tertiary care general hospital in Brazil. Nutr hosp. 2008;23(6):599-606.

16. Kac G, Dias PC, Coutinho DS, Lopes RS, Vilas-Boas V, Pinheiro AB. Length of stay is associated with incidence of in-hospital malnutrition in a group of low-income Brazilian children. Salud pública Méx. 2000;42(5):407-12.

Recebido: 04/4/2013

Aprovado: 25/5/2013 